\title{
Perception of Rotating Spiral Patterns by Pigeons
}

\author{
C. Martinoya ${ }^{1}$ and J. D. Delius ${ }^{2}$ \\ 1 Institut de Neurosciences, Université Pierre et Marie Curie/CNRS, 9 Quai St. Bernard, F-75005 Paris, France \\ 2 Allgemeine Psychologie, Universität Konstanz, Postfach 5560, D-7750 Konstanz 1, Federal Republic of Germany
}

\begin{abstract}
The ability of pigeons to discriminate indepth moving stimuli was studied with the rotating spiral illusion. Trained with tightly wound spirals, the birds were able to distinguish apparently approaching from apparently retreating spirals. Discrimination also persisted with loosely wound spirals, even though these did not induce an equivalent illusion in humans. Analysis of the optic flow created by the spirals indicates that the relevant cues were local divergent/convergent motion patterns. Global flow patterns, similar to those arising with approaching/retreating scenes, were only generated by tightly wound spirals. An unidimensional parameter $\tau$ could be derived that typified each and all the stimuli used. It is equivalent to the $\tau$ that has been used to characterize the optic flow of really approaching objects, indicating the time to collision. With a stationary rotating logarithmic spiral, $\tau$ is a joint function of winding tightness and rotation velocity. The $\tau s$ associated with the rotation speeds yielding threshold discrimination gauged the effectiveness of spirals with different winding inclinations. Threshold $\tau s$ were high with tight spirals and decreased with loose spirals. This indicates that both local and global kinetic cues must contribute to the detection of in-depth movement by pigeons. Even though the cue efficiency of local flow patterns alone is less than that of global flow patterns the former may be of value when they are dealing with scene elements looming at different rates or with looming objects that are partially occluded.
\end{abstract}

\section{Introduction}

Animals capable of moving at high speed can be expected to be efficient in perceiving movement in depth. Many fast moving species however cannot make much use of binocular stereoscopic cues for this purpose because their eyes are too close together to provide the essential image disparities or because the visual fields of the two eyes have little overlap. Such animals can be expected to rely mainly on the so-called optic flow patterns that are generated by approaching and retreating stimuli. Optic flow designates the overall kinetics of two-dimensional retinal images generated by relative motion between observer and surrounding scenes and objects. Approaching and receding stimuli respectively yield an expansion and a contraction of the retinal images. There is much evidence that even in species with good stereoscopic vision optic flow is an important alternative cue for the perception of movement in depth (Dodwell 1984; Gibson 1979; Graham 1968; Koenderink 1986; Lee 1976; Warren 1988). It has been shown that humans and some animal species respond to expanding (looming) images with reactions similar to those elicited by real approaching objects or scenes (Carpenter and Carpenter 1958; Schiff et al. 1962; Schiff 1965; Swanson and Gogel 1986).

To be able to make use of optic flow cues, organisms must possess neural mechanisms that can extract velocity vector arrays from dynamic retinal images. There is much evidence that the visual system of many species includes a profusion of neurons suitably responsive to visually moving stimuli (Frost 1986). Indeed, there is even evidence that certain neural units respond quite specifically to looming stimuli (Cynader and $\mathrm{Re}$ gan 1978; Rauschecker et al. 1987; Regan and Cynader 1979; Toyama et al. 1985).

The performance of visually guided behaviour has been related to the kinetics of retinal images in studies on humans (sports and driving: Lee 1976, 1980) and on animals (landing in flies: Braitenberg and Tadei-Ferretti 1966; Eckert and Hamdorf i980; Wagner 1982; Eckert 1983; diving in gannets: Reddish and Lee 1981). Important insights have also come from authors primarily concerned with the development of artificial vision systems (Hildreth 1984; Uras et al. 1988). In some ways, however, theory has raced ahead of empirical knowledge (Nakayama 1985). There are few psychophysical experiments providing insight into the processing of optic flow information. Most of these studies have dealt with humans (Beverly and Regan 1979; Lee 1976, 1980; 
Perrone 1986; Regan and Beverly 1978), a species where correlation with neurophysiology has limited prospects. The technical effort required has probably inhibited comparable experiments on animals.

In fact, an actual change in spatial location or image size is not required to induce the impression of in-depth movement. Stimuli of constant size presented at a constant distance whose features only appear to move outwards due ot kinetic induction phenomena are sufficient (Martinoya 1977). A relevant example is the expansion/approach illusion induced by a rotating spiral.

Many results relating to looming stimuli can be conveniently rationalized by introducing a so-called $\tau$ parameter. Given a certain organism-target distance $D$, $\tau$ specifies the time that would elapse until actual (or virtual) collision occurs, should the relative approach velocity $U$ remain constant. The time to collision is objectively given by $\tau=D / U$. However, $D$ and $U$ are not elements of the retinal image kinetics. Because of the projective properties of conventional eyes the expansion $1 / \tau$ happens fortunately to be equal to the image's relative rate of expansion velocity (RREV), the quotient between the velocity of expansion of the retinal image $\mathrm{d} a / \mathrm{d} t$ and its size a $(\operatorname{RREV}=(\mathrm{d} a / \mathrm{d} t) / a)$.

The present contribution will also refer to the parameter $\tau$. It begins, however, by reporting a behavioural experiment in which pigeons discriminated various spiral patterns rotating at controlled speeds. The results suggest that local as well as global kinetic cues contribute to the perception of in-depth movement by pigeons.

\section{Methods}

\subsection{Subjects}

Four adult domestic pigeons (Columba livia) of homing stock were employed. Three were experimentally naive, one had served in a previous experiment (see below). They were maintained at $80 \%$ of their normal body weights.

\subsection{Apparatus}

A Skinner-box $15 \mathrm{~cm}$ wide, $40 \mathrm{~cm}$ long and $40 \mathrm{~cm}$ high, painted matt white was used (Fig. 1). A bulb affixed to the ceiling provided illumination $\left(50 \mathrm{~cd} / \mathrm{m}^{2}\right.$; house light). Two transparent pecking keys $3 \mathrm{~cm}$ in diameter were arranged side by side ( $5 \mathrm{~cm}$ between centres) $20 \mathrm{~cm}$ above the floor on one of the narrow walls. The trough of an automatic food dispenser was located on the floor centred below the keys. A small light bulb ( $2 \mathrm{~W}$ ), immediately above, illuminated the trough (reward light).

Green and red paper discs behind the pecking keys served as stimuli during pretraining. For training and testing they were replaced by two white discs $(4 \mathrm{~cm}$ diameter) bearing black spiral drawings (see below). Each disc was mounted on an axle $(B, C)$ supported by

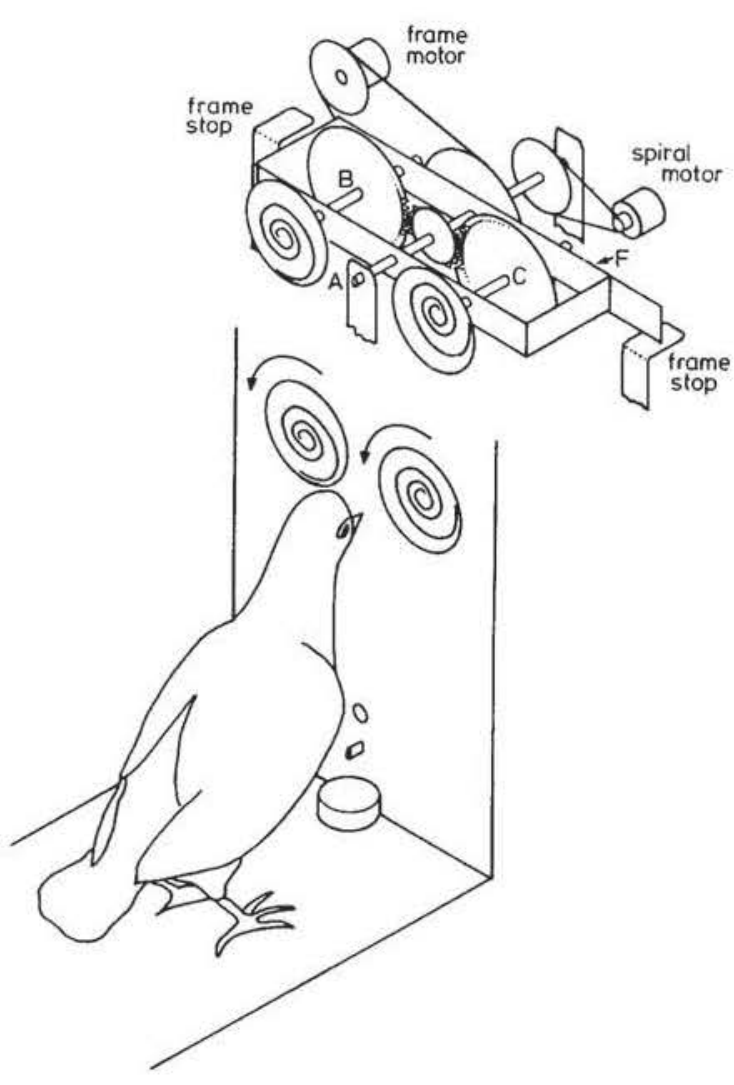

Fig. 1. A pigeon discriminating between rotating spirals displayed behind transparent response keys. The mirror image spirals rotate anticlockwise (arrows) in this instance. The left nonconformly rotating spiral induces an illusory expansion; the right spiral rotating conformly induces an illusory contraction. The upper drawing depicts the stimulus control mechanism (see text)

a metal frame $F$ (Fig. 1). A small, variable-speed electric motor drove both axles either clockwise or anticlockwise. Another motor permuted the right/left positions of the discs around axle $A$. White noise ( $50 \mathrm{db}$ SPL) masked ambient and apparatus noise. The apparatus was controlled by a microcomputer through an interface. A printer provided trial by trial protocols with block summaries. The animals' behaviour was monitored with video.

\subsection{Pretraining}

The pigeons were first taught to peck the keys to obtain a few grains of millet. Then they learned to discriminate the green and red discs where responses to green were rewarded and responses to red were not rewarded (the procedure was otherwise as described below). When they were yielding $75 \%$ correct choices after about 200 trials of this easy task the pigeons were transferred to the spiral discrimination task.

\subsection{Stimuli}

The stimuli were multi-branch rotating spirals. Logarithmic spirals were chosen because a rotation around 

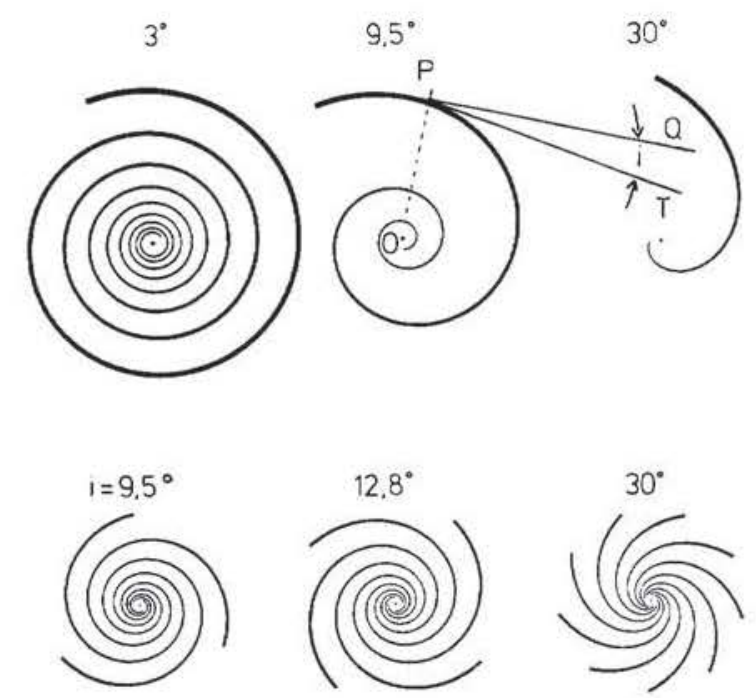

$45^{\circ}$

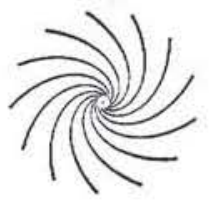

$60^{\circ}$

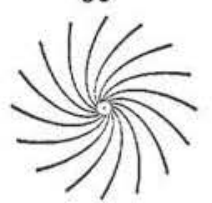

$90^{\circ}$

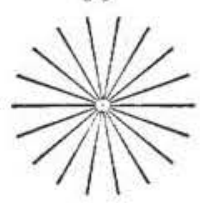

Fig. 2. Logarithmic spirals. Top: single branch, left wound spirals. The inclination $(i)$ is defined by the angle between the tangent $P T$ and the perpendicular $P Q$ to the radius $O P$ at point $P$. Low inclination spirals are tightly wound, high inclination spirals loosely wound. Bottom: multibranched spirals used as stimuli (some clockwise, some anticlockwise wound). The variable number of branches keeps the total line length constant. An $i=90^{\circ}$ spiral (lower right) degenerates into a pattern of radial spokes

the polar coordinate origin defining them is geometrically equivalent to an expansion with the origin as focus. They were drawn on the stimulus discs by computer. The polar coordinate equation of a single-branch logarithmic spiral (Fig. 2, see also Fig. 6) is $r=r_{0} * \exp (k * z)$ in which $r$ is the radius, $z$ is the angle, $r_{0}$ the radius for $z=0$, and $k$ is a constant influencing the tightness of the spiral's winding. This constant can be expressed as $k=\tan (i)$ where $i$ is the angle between the spiral's tangent $P T$ and the perpendicular $P Q$ to the radius $O P$ at a point $P$ of the spiral. The inclination angle $i$ fully characterizes a particular logarithmic spiral. Spirals can accordingly be described as low inclination (e.g. $i=9.5^{\circ}$ ), tightly wound or as high inclination (e.g. $i=60^{\circ}$ ), loosely wound. At $i=90^{\circ}$ a logarithmic spiral degenerates to a radial line.

Within a limiting circle a low inclination spiral describes more turns than a high inclination spiral. To compensate for the consequent line length differences the stimulus spirals had an increasing number of branches as their inclination rose (Fig. 2). For all spirals used the total branch length was the same $(230 \pm 5 \mathrm{~mm})$.

The stimuli that were used at any particular time were right (clockwise) wound and left (anticlockwise) wound, mirror-symmetric versions of a spiral of a given inclination. At any particular trial they were both

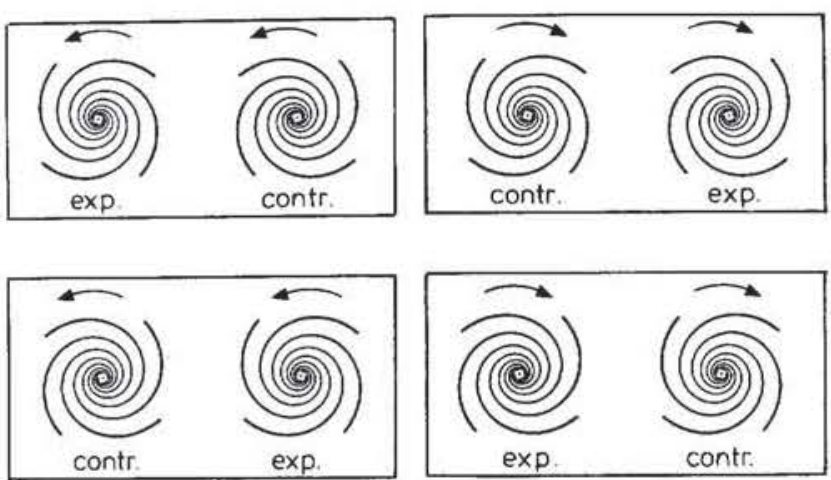

Fig. 3. Stimulus configurations employed. Left-wound/right-wound spiral pairs rotated clockwise or anticlockwise (arrows), the right side/left side being permuted. Within each configuration one stimulus induces an illusory expansion (exp.), the other an illusory contraction (contr.)

rotated clockwise or anticlockwise. In conjunction with the right/left permutation of the spiral versions there were 4 possible configurations (Fig. 3). Successive trials involved the presentation of all 4 in randomized order.

When tightly wound spiral is rotated in the same direction as it is wound (conform rotation) it is perceived by humans as a contracting (retreating) pattern. When the directions of winding and rotation are opposite (disconform rotation) the same spiral is perceived as an expanding (approaching) pattern. Assuming that the same would be true for pigeons the expanding or contracting quality of the stimuli was defined as positive (rewarded) or negative (non-rewarded) within each configuration. Neither left/right key, left/right spiral nor left/right rotation but only the conformity or disconformity of winding and rotation was correlated with reward/non-reward. "Contracting" stimuli were positive for subjects $C 16, C 19$ and $C 286$, "expanding" stimuli were positive for subject $X 21$.

In the course of the experiment each pigeon was trained to discriminate spirals of a given inclination rotated at a standard speed (see below) and then tested with the same spirals rotating at systematically decreasing speeds. This was repeated with spirals of various inclinations. Controls with degenerate $90^{\circ}$ spirals were interspersed at regular intervals.

\subsection{Training}

A simultaneous discrimination conditioning procedure with discrete trials was used. The stimulus configuration for a given trial was set up as the house light was switched off at the end of the previous trial. The light came on again after $3 \mathrm{~s}$. Three pecks to the key displaying the positive stimulus were rewarded with grain. The animals had $3 \mathrm{~s}$ to consume them while the house light was off and only the reward light was on. Three pecks to the key displaying the negative stimulus were punished with $3 \mathrm{~s}$ total darkness. If the subject did not respond within $10 \mathrm{~s}$ the trial ended and an omission was recorded. 
The stimulus configurations alternated randomly from trial to trial except that a given configuration was never used more than twice in succession. Within blocks of 16 testing trials each of the configurations occurred equally often. However, trials that had yielded erroneous choices were repeated with a probability of 0.5 . These correction trials were not scored. Performance within a block was assessed by the percentage of correct choices over the total choices (perfect discrimination: $100 \%$; chance discrimination: $50 \%$; omissions and correction trials disregarded). The number of blocks per session varied between about 10 and 20 . The sessions terminated prematurely when subjects produced 4 or more omissions during each of 3 successive blocks. Sessions were run daily except weekends.

The pigeons began by learning to discriminate $i=9.5^{\circ}$ spirals rotating conformly and disconformly at $\omega=1.5 \mathrm{arcrad} / \mathrm{s} \quad\left(\right.$ about $\left.90^{\circ} / \mathrm{s}\right)$. When the pigeons achieved $80 \%$ or more correct responses in 3 consecutive training blocks, they proceeded to the testing phase. The training (and testing) procedure was repeated with spirals of $12.8^{\circ}, 30^{\circ}, 60^{\circ}$ and $45^{\circ}$ inclination.

\subsection{Testing}

Each testing session started with warm-up blocks involving the standard rotation speed (1.5 $\mathrm{arcrad} / \mathrm{s})$. These ended when the subject attained a block score of $90 \%$ or better. The rotation speed for the following blocks was stepped down according to a descending progression $\left(\omega_{n+1} \approx \omega_{n} * 0.7\right)$ until a block score dropped to $65 \%$ correct or less. If the animal was still below $90 \%$ normal weight, testing then restarted at the standard rotation speed stage; if not the testing session ended. Performance was assessed as before, but blocks with 4 or more omission errors were ignored. If 2 or more blocks had to be excluded, the entire step-down sequence was discarded. By repeating sessions, between 150 and 250 valid test trials at a given rotation speed were accumulated for each bird. The same training/testing routine applied to each of the spiral inclinations mentioned above. Eventually, some 28,000 trials were available for evaluation.

\section{Results}

Video monitoring showed that the pigeons always fixated the stimuli frontally and statically before responding. As is well known (Delius 1985) they regularly also closed their eyes during the approach phase of the key peck. The spiral discrimination task proved to be comparatively difficult for naive pigeons. This may relate to the fact that looming stimuli do not normally control pecking in pigeons. The subjects that had only colour discrimination pretraining $(C 16, C 19$ and $X 21)$ needed more than 2000 trials to master the initial task. By contrast the pigeon $C 286$ needed less than 200 trials to reach criterion. In a previous, unpublished experiment it had learned to discriminate concentric circles that were either zoomed-up (positive) or zoomed-down (negative) in size. Tests had shown it to be the only one of 6 pigeons partaking in that experiment that had not relied on any artifactual cues technically associated with image contraction/expansion (size differences, luminosity changes, mechanical noises). The rapid acquisition in the present experiment indicates that this bird was able to transfer what it had learned previously. It presumably saw the rotating spirals as contracting and expanding much as it had seen the annular images actually zooming up or down. This strongly suggests that pigeons are subject to the rotating spiral illusion.

Switches between spirals of different inclination did not disrupt discrimination. In every case the criterion was reattained in less than 10 training blocks. This was also the case when the pigeons were suddenly presented with $60^{\circ}$ spirals after having only experienced spirals of up to $30^{\circ}$ inclination. This smooth transition was surprising because humans perceived these stimuli as patently different. The low inclination spirals evoked the illusion of size change and depth movement but the high inclination spirals were seen simply as rotating figures.

Test results were evaluated by plotting percent performance scores based on between 150 and 250 trials against the corresponding angular rotation speeds $\omega$, separately for different animals and spiral inclinations. Typical examples of the psychophysical curves so obtained are shown in Fig 4. The recurring and steady decrease in discrimination performance with diminishing $\omega$ shows that the animals' responses were under close stimulus control. Control tests with degenerate $90^{\circ}$ "spirals" invariably yielded scores that were indistinguishable from chance $50 \%$ performance. Artifactual, unintended cues therefore did not influence the animals' choice responses.
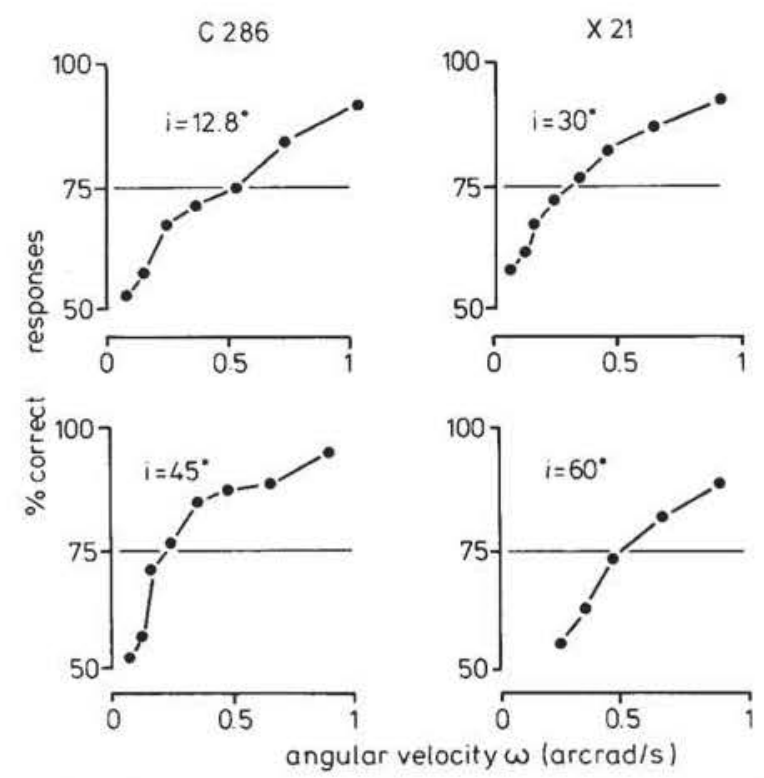

Fig. 4. Examples of psychophysical functions derived from test results (pigeons $C 286$ and $X 21$, spirals of various inclinations). Discrimination performance plotted as a function of angular rotation speed 


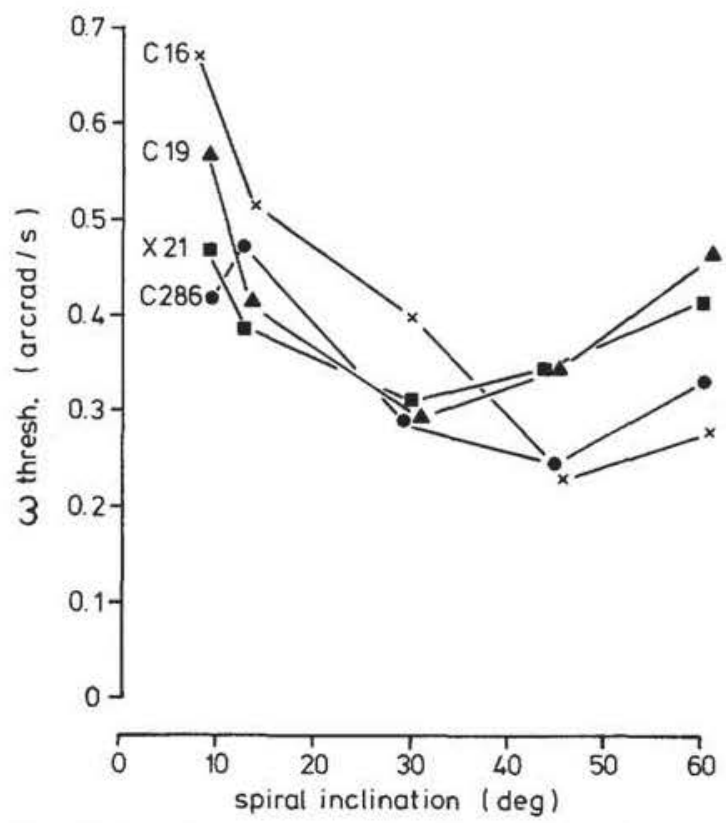

Fig. 5. Rotation speeds $\omega$ at threshold ( $75 \%$ choices correct) as a function of spiral inclination $i$ for 4 pigeons

Linear regressions were fitted to the performance scores as a function of $\log \omega$. The $\omega s$ corresponding to $75 \%$ correct discrimination $\left(\omega_{\text {thresh. }}\right)$ are plotted in Fig. 5 as a function of spiral inclination. Overall the curves evince a descending-concave, non-monotonic trend. It is obvious that the pigeons' discrimination performance depends both on the spirals' rotation speed and inclination. A more unified account of these results is developed below.

\section{Discussion}

\subsection{Optic Flow}

The optic flow patterns produced by the rotating spirals is likely to be the principal cue controlling the pigeons behaviour. An optic flow pattern is the two-dimensional array of local motion vectors associated with a timevarying image. It corresponds to the overall activation pattern of local movement detectors. Such detectors are found in many visual systems. Pearlman and Hughes (1976) for example described cells in the pigeon's retina each of which responded to linear movement within a small patch (receptive field) of the total visual field. The activity of these cells was a function of the stimulus velocity. Each of the cells was maximally responsive to movement in a particular direction. Every patch of the visual field was apparently monitored by a number of cells each having a different preferred direction.

Such movement detectors are affected by the socalled aperture problem (Hildreth 1984). When an extended line or bar moves across the receptive fields it is not necessarily the detectors tuned to the veridical direction that respond most. Rather it is those tuned to a direction perpendicular to the line orientation that react maximally. Movement detectors sensitive to point features can, however, respond veridically. But as the spirals used here consist mainly of extended lines it is only the effect of the former detectors that needs to be considered.

\subsection{Global/Local Flow}

Following upon the above argument the stimulus effective for local motion detectors with aperture $C$ at any point $P$ of a spiral branch $S$ will only be the component of the actual velocity $V$ of that point that is perpendicular to the branch's tangent $P T\left(V_{p}\right.$, Fig. 6). The activation of a given motion detector will in turn be related to the magnitude of the projection $V_{d}$ of the perpendicular component $V_{p}$ onto that detector's preferred direction $P D$. Still, among the ensemble of detectors "looking" at the relevant point $P$ those whose preferred direction come closest to $P P^{\prime}$ will be the most activated. More generally, the ensemble's overall response will be representative of the perpendicular motion vector with the magnitude $V_{p}=\omega * r * \sin (i)$. This applies to all points of the spiral. The optic flow that the rotating spiral creates correspond to the array of these vectors.

The optic flows induced by the disconform rotation of low and high inclination spiral are schematically depicted in Fig. 7 top. The optic flow patterns generated by conform rotation are analogous except for the opposite direction of the vectors. A tightly wound spiral

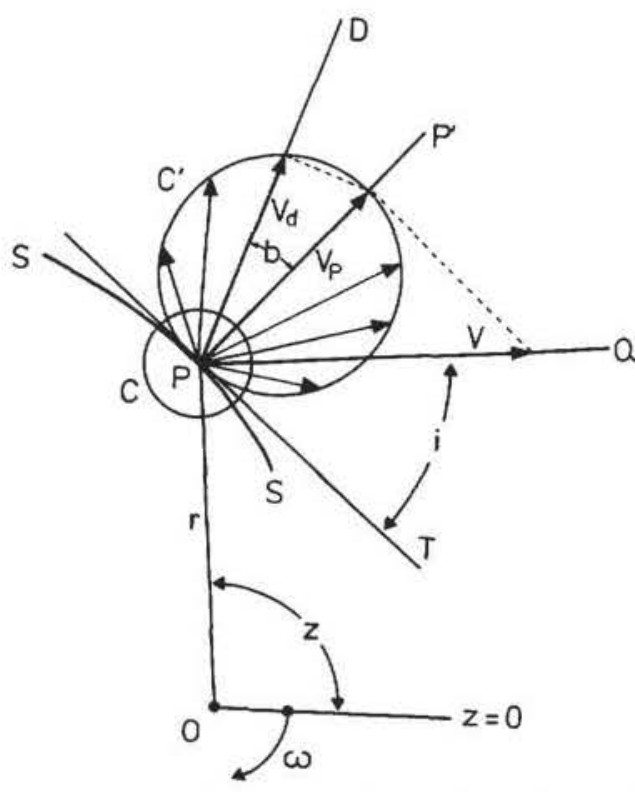

Fig. 6. Motion detected at a given point $P$ of a rotating spiral arm SS by movement detectors with preferred directions and a restricted aperture $C . O$ : origin of polar coordinates and center of rotation; $r$ : polar radius; $\omega$ : spiral rotation velocity; $P T$ : tangent to spiral at point $P$; $i$ : spiral inclination; $V$ : actual motion of point $P ; V_{p}$ : motion component in the direction $P P^{\prime}$ perpendicular to $P T ; P D$ : preferred direction of detector; $b$ : angle between $P D$ and $P P^{\prime} ; V_{d}$ : velocity detected by detector with preferred direction $P D ; C^{\prime}$ : circle defining velocities detected by ensemble of detectors with apertures centred on $P$ 



Fig. 7. Top: Schematic optic flows induced by rotating spirals of $9.5^{\circ}$ (left) and $60^{\circ}$ (right) inclination. Both spirals are wound anticlockwise and rotate clockwise (disconform rotation). The vectors depicted are the apparent instantaneous perpendicular motions $V_{p}$ of points evenly spaced along the spiral branches. The left flow is characterized by expansion, the right one by rotation. The insets are partial enlargements (see text). Bottom: Flows induced by an actually expanding concentric annular pattern (left ) and by a rotating pattern of spokes

gives rise to an optic flow quite different from the optic flow created by a loosely wound spiral. The former creates a flow similar to that produced by an expanding/approaching (looming) annular grid (Fig. 7 bottom left): all local motion vectors diverge from a central focus of expansion and the vector's length is in each case proportional to the distance of its origin to the center of rotation. The only difference is that in the case of the annular grid the constituent vectors are perfectly aligned with the polar radii while in the latter case they deviate by the (small) angle $i$ from the same radii.

Loosely wound spirals create an optic flow rather similar to that induced by rotating spokes (the degenerate $i=90^{\circ}$ spiral; Fig. 7 bottom right). The vector's lengths are still proportional to the eccentricity but their orientations diverge strongly from the corresponding radii (by the large angle $i$ ). In case of the spoke pattern all constituent vectors are perpendicular to the radii.

\subsection{Flow Commonality}

Despite the marked global differences between the flows generated by rotating low and high inclination spirals they share a pattern of diverging local vectors (see magnified detail inserts, Fig. 7). With low inclination spirals these local patterns are coherent with the global pattern because they approximately radiate from the spiral's center. In the case of high inclination spirals the vectors diverge from the local foci of branch curvature. Neural circuits collating flow information from restricted visual neighbourhoods will, however, in both instances extract analogous messages. The fact that pigeons continued to discriminate when switched from low to high inclination spirals indicates their choice behaviour was at least partially controlled by local flow detectors. The local/global distinction made here may relate to the small/large receptive fields that characterize different motion sensitive neurons at various levels of the pigeon's central visual system (Emmerton 1983). However, to our knowledge, these neurons have never been tested for the convergent/divergent motion sensitivity that is postulated here.

Incidentally, according to our experience humans can also distinguish conformly and disconformly rotating high inclination spirals as turning towards or against their curvature. They may thus also possess detectors discriminating between local convergent and divergent optic flows.

\subsection{Rate of Expansion}

A so-called relative rate of expansion velocity (RREV) measure is useful to characterize the optic flow of approaching scenes. As explained earlier it is a simple matter to derive RREV from a looming optic flow pattern. An expression for RREV can be similarly derived for low inclination spirals rotating disconformly. At a given point of a spiral the radius $r$ can be taken as a measure of the image size, and the projection $V_{r}$ of the perpendicular component $V_{p}$ onto the corresponding polar radius as the expansion velocity. This translates to $V_{r}=V_{p} * \cos (i)=\omega * r * \sin (i) * \cos (i)$ for the expansion velocity and hence to RREV = $V_{r} / r=\omega * \sin (i) * \cos (i)$ for the relative rate. Since, for a given logarithmic spiral, $i$ is constant, RREV is the same at all points of the spiral.

For high inclination spirals the corresponding relative rate of expansion velocity is given by RREV = $V_{p} / R$ in which $R$ is the local radius of curvature for a given point of the spiral. The latter is $R=r / \cos (i)$ and thus $\operatorname{RREV}=V_{p} / R=\omega * \sin (i) * \cos (i)$. This expression, obtained on the basis of a "local" analysis of the optic flow of high inclination spirals is identical to that deduced above on the basis of a "global" analysis of low inclination spiral flows.

\subsection{Time to Collision}

The most drastic event in connection with an approaching stimulus is bodily impact with it. It follows that animals should somehow be able to continuously assess the time to collision. $\tau$ is a parameter that provides that information and as explained in the Introduction is given by $\tau=1 /$ RREV.

With rotating logarithmic spirals $\tau=1 /(\omega *$ $\sin (i) * \cos (i))$. Normally $\tau$ is conceived as quantifying 


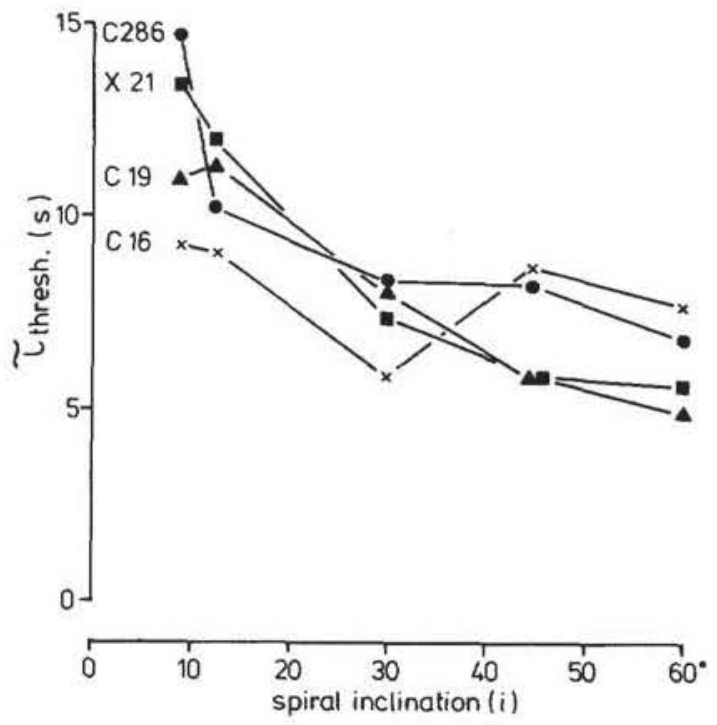

Fig. 8. Time to collision at threshold $\left(\tau_{\text {thresh. }}\right)$ plotted as a function of spiral inclination $i$. For three pigeons $(C 19, X 21$ and $C 286), \tau_{\text {thresh. }}$ decreases with increasing values of $i$. For the fourth pigeon $(C 16)$ there is no regular trend

the approach of expanding images (here disconformly rotating spirals) but it is feasible to derive the exact analogue parameter $\tau^{\prime}$ for contracting (receding) stimuli (here conformly rotating spirals) where it defines the time to double distance. Since pigeons in the present experiment discriminated between apparent expansion and apparent contraction both $\tau$ and $\tau^{\prime}$ are implicated. But as they are fully complementary the compound $\tau, \tau^{\prime}$ estimate can be expected to be a good estimator of $\tau$ itself.



Fig. 9. Average $\tau_{\text {thresh. }}$ (with standard errors) for pigeons $C 19, X 21$ and $C 286$ as a function of the spiral inclination $i . \tau_{\text {thresh. }}$ decreases monotonically as inclination increases $(r=0.84, p<0.005)$
Table 1. $\tau_{\text {mresh }}$ (with standard error) for spirals of different inclination

\begin{tabular}{lllccc}
\hline Pigeon & $i=9.5^{\circ}$ & $=12.8^{\circ}$ & $=30^{\circ}$ & $=45^{\circ}$ & $=60^{\circ}$ \\
\hline$C 286$ & 14,67 & 10.10 & 8.14 & 8.15 & 6.94 \\
& $(0.79)$ & $(0.57)$ & $(0.58)$ & $(0.67)$ & $(0.70)$ \\
$X 21$ & 13.31 & 12.10 & 7.38 & 5.82 & 5.57 \\
& $(0.71)$ & $(0.87)$ & $(0.52)$ & $(0.34)$ & $(1.49)$ \\
$C 19$ & 10.87 & 11.24 & 7.98 & 5.82 & 4.94 \\
& $(0.72)$ & $(1.01)$ & $(0.79)$ & $(0.44)$ & $(0.34)$ \\
$C 16$ & 9.16 & 9.01 & 5.79 & 8.73 & 7.68 \\
& $(0.97)$ & $(0.67)$ & $(0.36)$ & $(0.48)$ & $(0.57)$ \\
\hline
\end{tabular}

Entering the $\omega_{\text {thresh. }}$ values experimentally found for each bird and each spiral inclination into the above formula yields corresponding $\tau_{\text {thresh. }}$ values. They are listed in Table 1 and depicted in Fig. 8. It is apparent that for three birds $(X 21, C 19, C 286) \tau_{\text {thresh. }}$ is an inverse function of spiral inclination. The data of the remaining bird $(C 16)$ do not yield an orderly function and will be ignored in what follows. It can be seen (Fig. 9) that the average $\tau_{\text {thresh. }}$ of the others decrease from about $13 \mathrm{~s}$ to about $6 \mathrm{~s}$ as $i$ rises from $9.5^{\circ}$ to $60^{\circ}$ indicating a decreasing efficiency in collision forecasting as spiral tightness decreases. This is in agreement with the corresponding fall in global flow coherence discussed earlier. The result suggests that the pigeons' behaviour in our experiment was controlled weakly by local verging flow detectors and strongly by global verging flow detectors. Neurally, the activity of these latter detectors may be thought as selectively representing the sum activity of an ensemble of spatially distributed local detectors that possess globally congruent flow sensitivities.

\subsection{Motion Sensitivity}

It remains to consider how the visual motion sensitivities implied by the above models compare with more directly measured sensitivities. The $V_{p} \mathrm{~s}$ at threshold have to be converted to standard angular velocities. According to the video observations the eye-stimulus distance during stimulus fixation was typically $d \approx 5 \mathrm{~cm}$ (see also Hodos et al. 1985). The angular velocity expressed in $\%$ is given by $\mathrm{d} a / \mathrm{d} t=(180 / \pi) *$ $\arctan \left(V_{p} / d\right)=57.3 * \arctan (\omega * \sin (i) / d)$ for any point of the spiral. The $r$ of the spirals used grew from $0.2 \mathrm{~cm}$ to $1.5 \mathrm{~cm} ; 1 \mathrm{~cm}$ will serve as a typical value. Inserting the average $\omega_{\text {thresh. }}$ of $0.48 \mathrm{arcrad} / \mathrm{s}$ for $9.5^{\circ}$ spirals and $0.35 \mathrm{arcrad} / \mathrm{s}$ for $60^{\circ}$ spirals yields threshold angular velocities of about $1 \%$ and $3.5 \%$ s. In a simple translation discrimination study Martinoya et al. (1983) obtained a threshold of $5.8 \%$ for frontally viewing pigeons, in close agreement with measurements by others (Emmerton 1983). Even though strict comparison is not feasible because of several procedural differences, the threshold velocity implied by the present data is markedly lower. Possibly detector mechanisms dealing with vergent motion, due to summation, achieve a greater sensitivity than more elementary ones tuned to simple translation movements. 


\section{Conclusion}

The behavioural results suggest that pigeons are subject to the expansion/contraction illusion induced by rotating spirals in a similar manner as humans, even though the two species have quite different visual systems (mainly tectum and cortex based respectively). The theoretical analysis furthermore indicates that purely visuo-kinetic cues (optic flow) are important for the detection of movement in depth by pigeons. They also suggest that, in accordance with ecological demands, these birds possess both local and global detectors for expanding and contracting optic flow. That their looming flow sensitivity is excellent is suitably illustrated by the fact that according to our findings (best $\tau_{\text {thresh. }} \approx 13 \mathrm{~s}$ ) a pigeon should be able to detect a predator approaching at say $40 \mathrm{~km} / \mathrm{h}(U \approx 11 \mathrm{~m} / \mathrm{s})$ when it is still about $140 \mathrm{~m}$ away $\left(D=\tau_{\text {thresh. }} * U\right)$. Further psychophysical studies with (technically expensive) truly looming stimuli are however still required for a more complete understanding of the optic flow perception by pigeons.

Acknowledgements. The research was partly carried out while C.M. was a guest of and J.D.D. was on the staff of the Psychologisches Institut, Ruhr-Universität, Bochum. The work was aided by a European Science Foundation Twinning Grant, a Deutsche Forschungsgemeinschaft Research Grant, a Heinrich Hertz Stiftung Fellowship and a Visiting Professorship, Universität Konstanz. We thank the Bochum and Konstanz lab members for much, varied and good-humored help. Dr. S. Bloch, Paris contributed with dynamic friendship, S. Ziffus, Konstanz with administrative know-how, Dr. H. Glünder, Munich with some insights, Dr. J. Emmerton, Bochum and J. Delius, Frankfurt with considered comment and improved English.

\section{References}

Beverly KE, Regan D (1979) Visual perception of changing size: the effect of object size. Vision Res 19:1093-1104

Braitenberg V, Tadei-Ferretti C (1966) Landing reaction of Musca domestica. Naturwissenschaften 55:155-156

Carpenter B, Carpenter JT (1958) The perception of movement by young chimpanzees and human children. J Comp Physiol Psychol 51:782-784

Cynader M, Regan D (1978) Neurons in cat's parastriate cortex sensitive to the direction of motion in three-dimensional space. $\mathbf{J}$ Physiol (London) 274:549-569

Delius JD (1985) The peck of the pigeon: free for all. In: Lowe CF, Richelle M, Blackman DE, Bradshaw CM (eds) Behaviour analysis and contemporary psychology, Erlbaum, New York, pp 53-81

Dodwell PC (1984) The Lie transformation groups model of visual perception. Percept Psychophys 34:1-16

Eckert H (1983) On the landing response of the blowfly, Calliphora erythrocephala. Biol Cybern 42:119-130

Eckert H, Hamdorf K (1980) Excitatory and inhibitory responses components in the landing response of Musca domestica. J Comp Physiol 138:273-276
Emmerton J (1983) Vision. In: Abs M (ed) Physiology and behaviour of the pigeon. Academic Press, London, pp 245-266

Frost BJ (1986) Motion characteristics of single units in the pigeon optic tectum. Vision Res 16:1229-1234

Gibson JJ (1979) The ecological approach to visual perception. Houghton Mifflin, Boston

Graham CH (1968) Depth and movement. Am Psychol 23:18-25

Hildreth EC (1984) Computations underlying the measurement of visual motion. Artif Intell 23:309-354

Hodos W, Bessette BB, Macko KA, Weiss SRB (1985) Normative data for pigeon vision. Vision Res 25:1525-1527

Koenderink JJ (1986) Optic flow. Vision Res 26:161-180

Lee DN (1976) A theory of visual control of braking based on information about time-to-collision. Perception 5:437-459

Lee DN (1980) Visuo-motor coordination in space-time. In: Stelmach GE, Requin J (eds) Tutorials in motor behavior. North Holland, Amsterdam, pp 281-295

Martinoya C (1977) Kinetic induction produced by light stimulation in humans. Vision Res 17:598-608

Martinoya C, Rivaud S, Bloch S (1983) Comparing frontal and lateral viewing in the pigeon. II. Velocity thresholds for movement discrimination. Behav Brain Res 8:375-385

Nakayama T (1985) Biological image motion processing; a review. Vision Res 25:625-660

Pearlman AL, Hughes CP (1976) Functional role of efferents to the avian retina. I. Analysis of retinal ganglion cell receptive fields. J Comp Neurol 166:111-122

Perrone JA (1986) Anisotropic responses to motion towards and away from the eye. Percept Psychophys 39:1-8

Rauschecker JP, Grünau MW von, Poulin C (1987) Centrifugal organization of direction preferences in the cat's lateral suprasylvian visual cortex and its relation to flow field processing. J Neurosci 7:943-949

Reddish PE, Lee DN (1981) Plummeting gannets: a paradigm of ecological optics. Nature 293:293-294

Regan D, Beverly KE (1978) Looming detectors in the human visual pathway. Vision Res 8:415-421

Regan D, Cynader M (1979) Neurons in area 18 of cat visual cortex selectively sensitive to changing size: nonlinear interaction between responses to two edges. Vision Res 19:699-711

Schiff W (1965) Perception of impending collision. Psychol Monogr 79:1-26

Schiff W, Cavines JA, Gibson JJ (1962) Persistent fear responses in rhesus monkeys to the optical stimulus of "looming". Science 136:982-983

Swanson MT, Gogel WC (1986) Perceived size and motion in depth from optical expansion. Percept Psychophys 39:309-326

Toyama K, Komatsu Y, Kasai H, Fujii K, Umetani K (1985) Responsiveness of Clare-Bishop neurons to visual cues associated with motion of a visual stimulus in three-dimensional space. Vision Res 25:407-414

Uras S Girosi F, Verri A, Torre V (1988) A computational approach to motion perception. Biol Cybern 60:79-87

Wagner H (1982) Flow-field variables trigger landing in flies. Nature 297:147-148

Warren WH Jr, Hannon DJ (1988) Direction of self-motion is perceived from optic flow. Nature 336:162-163

Prof. Dr. J. D. Delius

Allgemeine Psychologie

Universitāt Konstanz

Postfach 5560

D-7750 Konstanz

Federal Republic of Germany 